\title{
Determining whether age retirement for NBA players is associated with injuries and illnesses: A retrospective study
}

\section{Po-Hsin Chou}

National Yang-Ming University

Yu-Tsen Yeh

Geoger's University of London

CHIEN TSAI WEI ( $\square$ rasch.smile@gmail.com )

Chi Mei Medical Center https://orcid.org/0000-0003-1329-0679

Shih-Bin Su ( $\nabla$ shihbin1029@gmail.com )

Chi Mei Medical Center

Research article

Keywords: retirement age, NBA, all-star player, social network analysis, bootstrapping method

Posted Date: July 23rd, 2020

DOI: https://doi.org/10.21203/rs.3.rs-42145/v1

License: (c) (i) This work is licensed under a Creative Commons Attribution 4.0 International License.

Read Full License 


\section{Abstract}

Background: The careers of professional sport players are usually influenced by injuries and skills. The careers of all-star MBA and regular players depend on their health and how rapidly their skills diminish with age, respectively. Whether the above phenomena are considered and supported in the National Basketball Association (NBA) requires verification.

Methods: We downloaded injury data from the NBA website in 2010-2018 and extracted all injury events $(=9,783)$ involving 779 NBA players. Among these players, 705 were retired, 236 possessed all-star titles (60 injured and 170 non-injured), and 466 were regular players (408 injured and 58 non-injured) from 2010 to 2018. Both samples of retired all-star $(n=236)$ and regular players $(n=466)$ were compared to verify the effect of injury and illness on the retirement age using the bootstrapping method to estimate $95 \%$ confidence intervals in medians. We hypothesized that injuries and illnesses are associated with the retirement age of all-star players and irrelative to that of regular NBA players. Social network analysis(SNA) was performed to highlight the top three injuries with the highest degree centralities in their respective clusters.

Results: We observed that the top three degree centralities include (1) sprained a left ankle, (2) sprained a right ankle, and (3) illness. The top two most injured NBA teams in 2010-2018, as obtained by the metrics for measurement, were Timberwolves $(x$-index $=32.86)$ and Hornets $(x$-index $=31.42)$. Most of the careers of NBA all-star players were affected by injuries/illnesses. The NBA careers of regular players were terminated mostly according to their skill.

Conclusion: Although a number of things can be debated with regard to NBA, everyone can agree on one thing: Injuries are the worst. We confirmed that injuries and illnesses exhibit significant associations with the retirement age of all-star players and are irrelative to that of regular NBA players. Sports medicine is urgently needed to help players in preventing possible injuries while in the court in the discernible future.

\section{Highlights}

- The National Basketball Association (NBA) is a popular sport around the world. The issue of injuries and illness associated with the retirement ages only on all-stars remains unknown and worthy to study.

- The traditional way to disclose the most frequent occurrences is to count them. The modern social network analysis considering the relationship between entities has not been used for reporting the most influent injuries/illness in NBA players, particularly applying visual dashboards onto Google Maps.

- The bootstrapping method used for estimating $95 \%$ confidence intervals was applied to compare differences between groups. All our research process was included in the Additional files that can help readers mimic the method used in the discernable future. 


\section{Background}

The nature of playing basketball has changed dramatically over the years, evolving from a game of finesse to a collision sport; it is currently designated as a high-risk contact sport [1]. The National Basketball Association (NBA) teams have been allowed to increase the total number of team players from 12 to 15 since 2006. A study [2] addressed the (1) increased rate of injuries in NBA until the expansion of team size in 2006, whereas a significant inverse association between games was missed due to injuries/illnesses and percentage of games won. Team expansion became positively associated with decreased injury/illness rates after 2006 [3]. Playing back-to-back games and away games are also significant predictors of frequent game injuries in the study [3].

\subsection{Association between injury/illness and retirement age}

Injuries impact the playing career and quality of life after the retirement of professional basketball players [4]. NBA all-stars, such as Dwyane Wade (37 years old in 2018), Yao Ming (31 years old in 2011), and Grant Hill (41 years old in 2013), retired at different ages.

As the total number of team players increased from 12 to 15 since 2006, changes in retirement age must be studied. However, the most important topic may be the injuries and illnesses influencing retirement age.

\subsection{Injury and skill affect the careers of NBA players}

NBA playing careers are usually influenced by injuries and skills. Whether NBA all-star and regular players are affected by injury and skill, respectively, should be studied. Thus, we hypothesize that injuries and illnesses are associated with the retirement age of all-star players but irrelative to that of regular NBA players.

\subsection{Types of injury and illness of NBA players}

Although a number of things can be debated in NBA, everyone can agree on one thing: Injuries are the worst. The top five NBA careers ruined by injuries were those of Derrick Rose, Grant Hill, Yao Ming, Brandon Roy, and Greg Oden [5]. Reportable injuries comprise those that result in (1) physician referral, (2) a missed practice or game, or (3) rendered emergency care [1]. Ankle sprain was the most frequently occurring orthopedic injury, followed by patellofemoral inflammation, lumbar strain, and knee sprain, as reported in 2000 [1]. Another study in 2010 [5] stated that lateral ankle sprain was the most frequent orthopedic injury, followed by patellofemoral inflammation, lumbar strain, and hamstring strain [6]. The latest analysis of injuries of NBA player from 2010 to 2018 [7] released the top four injuries, including sprained left ankle (DNP), rest (DTD), sprained right ankle (DNP), and illness (DTD), where DNP denotes "did not play," and DTD represents "day-to-day" rest due to other reasons.

Any change in the most frequently occurring injury should be inspected, particularly by using social network analysis (SNA) $[8,9]$, focusing on the associated clusters, instead of the traditional method, which 
only observes the frequencies of injuries and illnesses alone. For instance, Shaquille O'Neal, while playing for the Celtics, suffered from a sore right calf and sore left knee (2010/12/9), sore right calf and sore right calf $(2010 / 12 / 15)$, hip injury and hip injury $(2011 / 1 / 21)$, and others, which will be included in a related cluster highlighted by a representative, with the most number of degree centralities belonging to a specific independent cluster, unlike the traditional counting method for selecting the most frequent occurrences in a whole system.

In addition, impacts of injury on players or teams are often inspected by the total number of events using the traditional method. Whether author impact factors (AIF) or other relevant indicators in scientometrics can be applied to professional players requires investigation.

\subsection{Objectives}

In this study, we hypothesized that injuries and illnesses are associated with the retirement age of NBA all-star players and irrelative to that of regular players. The following tasks were implemented: (1) collection of injury data from the NBA website; (2) reporting of the most frequent injuries and players' age in relation to the injury frequencies; (3) visualization of the most injured NBA teams and players; (4) comparison of the differences in metric and retirement ages between two conditions (i.e., injury and noninjury) in two sample groups (i.e., all-star and regular players).

\section{Methods}

\subsection{Injury data from the NBA website}

We downloaded the injury data in 2010-2018 [10] from the NBA website on June 20, 2019, and extracted all transactions and events $(=9,783)$, including those of 779 NBA players. Among the players, 705 were retired, 236 had an all-star title (60 injured and 170 non-injured), and 466 were regular players (408 injured and 58 non-injured from 2010 to 2018). Both samples of 236 retired all-star players and 466 retired regular players were employed to verify the effect of injury and illness on the retirement age (see data deposited in Additional File 1). The team Bobcats was renamed as Hornets to let the number of NBA teams be 30 .

As the rest events (e.g., peak in April and not due to injury) were largely explained by teams heading to playoffs and resting players before the start of the game, we removed items with rest by DTD or DNP from the data.

We created a Microsoft Excel visual basic application module to handle the data. All downloaded data met the requireme nts for analyzing information from public websites. Ethical approval was not necessary for this study, as no human subjects nor personal data were accessed.

\subsection{Frequent injuries and player age in relation to the count frequencies}

\subsubsection{Results obtained by the traditional method}


The top 20 frequent injuries and illnesses and the players' ages in relation to the total counts of injuries and illnesses were displayed using the traditional method.

\subsubsection{Results obtained by a modern method (SNA)}

The injuries and illnesses associated with individual players were assigned to one record. Specifically, all injuries and illnesses experienced by the players were used to create clusters, and SNA was used for separation. The clustered injuries and illnesses were gathered in a group. On the other hand, the distantly related injuries and illnesses were partitioned further. SNA, we can examine the representative for each cluster that denotes the most influential in the respective cluster according to the number of degree centralities (i.e., the connections with other entities).

\subsection{Most injured NBA teams and players}

\subsubsection{Most injured NBA teams determined by using $x$-index}

The most injured NBA teams were selected by using $x$-index [11] in the bibliometric analysis. The $x$-index is defined by the formula $\left(=\sqrt{ } \max \left(c^{*} k\right)\right)$, where $k$ denotes the type of injuries in a team, and ci refers to the total number of each injury type. Similar to the citation analysis, the injury type was referred to as the number of publications for an author. The total count for each injury type was directed to the number of citations for an article (=the injury type). As such, the x-index and other indices (e.g., h, g, and Ag) [12,13] can be computed in MS Excel. Interested readers are invited to check out the computation process in Additional File 2.

\subsubsection{Most injured players determined by using $x$-index}

All 779 players, including retired and active NBA players, were included to present the extent of injury cases on a dashboard. Worst NBA injuries of the 2018-2019 season are shown in Additional File 3. Similar to the previous section discussing the most injured NBA teams, the $x$-index can be computed for each player.

\subsubsection{Visualizing NBA teams and players on dashboards}

We plotted the NBA teams and players on dashboards (see Additional File 2). The ci of $x$-index lies on the $Y$-axis, and the injury count at $k$ of $x$-index is on the X-axis for each player (or NBA team) on a map. The bubbles were sized by $x$-index, with a larger mean indicating more injuries/illnesses in the past. The bubbles were colored by groups (i.e., all-star players: green and regular players: yellow; or East conference: green and West Conference: yellow). Quick-response codes were created for readers, who can scan the codes on figures to obtain more information on dashboards.

\subsubsection{Computation of the bibliometric indices for each NBA team and player}

The indices were computed following the procedures below: 
- $x$-index [11]: All injury types were sorted first, and the maximum area was calculated by multiplying the number of injury types $(=k)$ and respective frequent observations at $k(=c i * k)$. Finally, the root of the rectangle was computed $\left(=x=\sqrt{ } c^{*} k\right)$.

- h-index [12]: All injury types were sorted first, and the maximum area was calculated by multiplying the number of injury types $(=k)$ and respective frequent observations at $k\left(=c i{ }^{*} k\right)$ based on $\mathrm{k} \leq \mathrm{ci}$. Finally, the root of the squared box $\left(h=\sqrt{ } k^{*} k\right)$ was computed.

- g-index [13]: All injury types were sorted first, and the most frequent accumulative observations were divided by the number of injury types at $\mathrm{g}$ ( $=\max \mathrm{Ag} / \mathrm{g}$ or squared $\mathrm{g}=\mathrm{Ag}$ ).

- Ag-index [13]: As described in the previous section, Ag denotes the frequent accumulative observations at a specific number of injury types $(=\mathrm{g})$.

\subsection{Differences in the retirement age between two conditions of injuries in two player groups}

We performed the bootstrapping method [14] to verify the differences in metrics and retirement age between two injury conditions (i.e., injury and non-injury) in two groups (i.e., all-star and regular players). A total of 1,000 median metrics were retrieved from the random samples, with 100 repetitions of median values for each metric and cluster. The median and $95 \%$ confidence interval $(\mathrm{Cl})$ were obtained to compare differences in the metrics and retirement age among groups by inspecting whether two $95 \% \mathrm{Cl}$ bands were overlaid.

\section{Results}

\subsection{Task 1: Relationship between the frequency of injuries and players' retirement age}

\subsubsection{Results obtained by the traditional method}

Table 1 shows the 20 most frequent injuries and illnesses. We observed that sprained left ankle, sprained a right ankle, and illness occupied the top three positions. Notably, numerous events without a cluster number in the last column in Table 1 were ruled out, indicating that they were negligibly important and involved in another relevant cluster using SNA for discrimination.

Figure 1 shows the frequency distribution of injuries/illnesses across different ages. The ages 31,34 , and 33 years old were associated with the three most frequently occurring injuries/illnesses.

\subsubsection{Results obtained using SNA}

Figure 2 shows the separation of several injury/illness clusters. The top three injuries with the greatest number of degree centralities obtained using SNA included (1) sprained left ankle, (2) sprained a right ankle, and (3) illness, similar to the results in Table 1. The differences from Table 1 were (1) the looser relation between injuries and those of another cluster; (2) the associated items that were gathered in one cluster, such as the clinical diagnosis-relative groups (DRGs) obtained using the concept of relationship 
to interpret the entities and their association with others. Interested readers are invited to scan the QRcode in Figure 2 and see details for a specific injury of interest.

\subsection{Task 2: Most injured NBA teams and players}

\subsubsection{Most injured NBA teams and the differences in metrics}

Timberwolves (West Conference) was the most injured NBA team with $\mathrm{x}$-index $=32.86(=\sqrt{ }$ frequency multiplied by the number of injury types $=\sqrt{ } 9 * 120)$. Hornets (East Conference) was the second most injured NBA team with $x$-index $=31.42(=\sqrt{ } 7 * 141)$ (see the top panel in Figure 3 ). Interested readers are suggested to scan the QR-code in Figure 3 to check out the other NBA teams and their indices.

Comparisons of metrics showed that the East Conference featured higher index values compared with the West Conference (see the bottom image in Figure 3).

\subsubsection{Most injured players and the differences in metrics}

The regular player Nate Robinson (Thunder, Warriors, and Nuggets) was the most injured NBA team player with $x$-index $=18.49(=\sqrt{ } 19 * 18=$ the maximal square root of frequency multiplied by the number of injury types). The all-star NBA player Kevin Love (Timberwolves and Cavaliers) was the second most injured NBA team player with $x$-index $=15(=\sqrt{ } 15 * 15)$ (see the top panel in Figure 4$)$. Interested readers are suggested to scan the QR-code in Figure 4 to check out the other NBA team players and their indices.

Comparisons of metrics showed that all-star players presented higher indices than the regular ones (see the bottom image in Figure 4).

\subsection{Task 3: Different retirement ages between two injury types in two player groups}

Table 2 presents the comparison of the retirement ages between groups using the bootstrapping method. The retirement age was only associated with the all-star group $(p<0.05)$, indicating that most NBA careers of all-star players were affected by injuries/illnesses. The NBA careers of regular players were possibly terminated by their skills in court.

\section{Discussion}

\subsection{What this knowledge adds to what we already know}

Ankle sprain was the most frequently occurring orthopedic injury in the past $[1,6]$, similar to the finding of this study (Table 1), which was obtained by the frequency count method. When SNA was applied, an identical result was obtained, similar to the result in Figure 2.

The curious reader may gain awareness of the difference between the traditional counting approach and SNA when referring to Table 1 and Figure 2. The themes shown in the latter might be clearer and more structural than the former. 
Injuries and illnesses affect players in terms of their sport careers and quality of life after retirement [4]. Retirement age depends on factors, such as health, skill, and love for career. All-star players show great charisma, which can prolong their NBA careers compared with the regular players.

Most large men, including Yao Ming and Amare Stoudemire, besides those who incurred injuries on their large bodies, can competitively play until their late 30's. Recently, for example, Tim Duncan, who retired at the age of 40 years old after having declined from his prime playing days, contributed well to his teams and also won a championship at the age of 37 . However, his body started to decline considerably, preventing him from competing against spry 20-year-old center players, such as DeMarcus Cousins (New Orleans Pelicans), Rudy Gobert (Utah Jazz), Karl-Anthony Towns (Minnesota Timberwolves), and Nikola Jokic (Denver Nuggets).

We removed 470 coded events from this study to obtain less relation to injuries and illnesses. Also, teams were resting players, and it was said to protect their stars healthy on the court or prepare for heading into playoffs. Coach Pop of Spurs rested his players the most in NBA. Teams may also rest players to increase their chances of finishing lower and having more balls in the lottery [7]. Interestingly, the results of injuries/illness in the NBA might be contaminated if resting players were analyzed in this study.

\subsection{What this study contributes to current knowledge}

According to the total number of injury events of the traditional method, the Bucks, Timberwolves, and Lakers have had the greatest number of injuries, whereas the Thunder, Blazers, and Pacers showed the least [7]. Kevin Love, Jason Smith, and Eric Gordon appeared on the list of players most frequently impacted by injuries [7].

If we consider the weights of the most games missed on injury types, including patellofemoral inflammation, lateral ankle sprain, knee sprain, and lumbar strain [6], the ranks of the most injured NBA teams and players will change. The determination of the most injured teams and players and the calculation of their metrics would be more complicated and difficult.

The total number of injury events (=sum(ci), where i ranges from 1 to $n$ injury types) can be compared with the number of citations in scientometrics. Several studies applied the total number of citations (=sum(ci), where i ranges from 1 to $\mathrm{n}$ on articles) to determine individual research achievements (IRA). AIF, similar to the total counts of injuries divided by the number of injury conditions suffered by players, has been used on IRA [15]; author-level metrics (e.g., $x, h, g$, and Ag) $[12,13]$ were proposed and applied in the literature $[8,9,16-19]$ to remove largely redundant citations and publications [20]. Accordingly, we attempted to apply scientometrics to evaluate the most injured NBA teams and players in Figures 3 and 4 , respectively. Hopefully, more discussions on the issue of using scientometrics in assessing the extent of the impact of injuries are expected in the future.

We also applied the bootstrapping method to estimate standard errors in data. As of June 20,2019, more than 227 articles were searched by the keyword "bootstrapping" in titles. The latest article [21] addressed 
the bootstrapping method that is suitable for use in time-to-event data, similar to the current study in Table 2, which compares the differences in retirement ages between groups (i.e., injury and non-injury).

\subsection{Implications of the results and suggested actions}

This study has several strengths. First, we applied SNA to objectively separate the clusters, which were rarely observed in previously published papers using SNA to evaluate injury/illness types in NBA. NBA allstar players and teams were shown on Google maps with dashboards, which can be manipulated by users to check the details on their own.

Second, the total number of injuries and illnesses for each type in Table 1 can be expressed by scientometrics, with $\mathrm{h}=35, \mathrm{x}=38.99, \mathrm{~g}=67$, and $\mathrm{Ag}=67.34$ (see Additional file 1 for details about the calculations). Referring to the relative weights (RWs) on DRGs, the physician's case mix index (CMI) is traditionally computed by the total RWs divided by the case number, similar to the AlF that was defined in a previous section. Whether scientometrics can be applied to evaluate individual $\mathrm{CMI}$, as we did on injured NBA teams and players, requires discussions in the future.

Third, the comparison between groups using the bootstrapping method is suitable for a data distribution fee, different from t-test and analysis of variance that requires normally distributed data.

Furthermore, we presented the research results with dashboard-type visual representations on Google maps, which were seldom observed in previous studies. The application of this animated display allows readers to easily and quickly browse more information on the internet.

\subsection{Limitations and suggestions}

This study features several limitations. First, caution should be exercised when interpreting and generalizing findings beyond the period from 2010 to 2018 of research as the data were extracted from the transaction events of an NBA-related website [10].

Second, the most injured NBA players were less meaningful in comparison because the results depending on player career were included in the data collection period. For instance, the NBA playing career for Nate Robinson and Kevin Love in Figure 4 might be longer than that of the others.

Third, the suitability of applying scientometrics to evaluate NBA teams and players impacted by injuries and illness should be discussed and examined further in the future because no such kinds of metrics have been applied to NBA nor other relevant fields besides bibliometric field.

Finally, the results in Table 2 cannot be generalized to other professional sports, such as the Major League Baseball, the National Football League, and the National Hockey League. The approaches applied in this study are recommended for other relevant research in the future.

\section{Conclusion}


There is plenty of room for debate in the NBA, but everyone can agree on one thing: Injuries are the worst. We confirmed that injuries and illnesses have significant associations with the retirement age of NBA allstar players and are irrelative to that of regular players. Sports medicine is in urgent need to help players in preventing possible injuries while playing in court in the discernable future.

\section{Declarations}

\section{Ethics approval and consent to participate}

Not applicable.

All data were downloaded from the website database at nba.com.

\section{Consent to publish}

Not applicable.

\section{Availability of data and materials}

All data used in this study is available in SDC files.

\section{Competing interests}

The authors declare that they have no competing interests.

\section{Funding}

There are no sources of funding to be declared.

\section{Authors' Contributions}

PH developed the study concept and design. TWC, YT and PH analyzed and interpreted the data. SBS monitored the process of this study and helped in responding to the reviewers' advice and comments. TWC drafted the manuscript, and all authors provided critical revisions for important intellectual content. The study was supervised by SBS. All authors read and approved the final manuscript.

\section{Acknowledgments}

We thank Enago (www.enago.tw) for the English language review of this manuscript. All authors declare no conflicts of interest.

\section{Abbreviations}

AlF= author impact factors 
$\mathrm{Cl}=$ confidence interval

$\mathrm{DNP}=$ did not play

DTD=day-to-day

NBA=National Basketball Association

SNA $=$ social network analysis

\section{References}

1. Starkey C. Injuries and illnesses in the national basketball association: a 10-year perspective. J Athl Train. 2000;35(2):161-7.

2. Podlog L, Buhler CF, Pollack H, Hopkins PN, Burgess PR. Time trends for injuries and illness, and their relation to performance in the National Basketball Association. JSci Med Sport. 2015;18(3):278-82.

3. Teramoto M, Cross CL, Cushman DM, Maak TG, Petron DJ. WillickSE. Game injuries in relation to game schedules in the National Basketball Association. JSci Med Sport. 2017;20(3):230-5.

4. Ekhtiari S, Khan M, Burrus T, Madden K, Gagnier J, Rogowski JP, Maerz T, Bedi A. Hip and Groin Injuries in Professional Basketball Players: Impact on Playing Career and Quality of Life After Retirement.Sports Health. 2019 May/Jun;11(3):218-222.

5. Hughes GThe 5 Best NBA Careers Ruined by Injuries.2019/6/20 retrieved at https://bleacherreport.com/articles/2795270-the-5-best-nba-careers-ruined-by-injuries\#slide5.

6. Drakos MC, Domb B, Starkey C, Callahan L, Allen AA. Injury in the national basketball association: a 17-year overview.Sports Health. 2010 Jul;2(4):284-90.

7. NBA Player Injuries from 2010 to 2018

Zivkovic J. Analyzing. NBA Player Injuries from 2010 to 2018. 2019/6/20 retrieved at https://www.kaggle.com/jaseziv83/extensive-nba-injuries-deep-dive-eda/comments.

8. Chien TW, Chang Y, Wang HY. Understanding the productive author who published papers in medicine using National Health Insurance Database: A systematic review and metaanalysis.Medicine (Baltimore). 2018 Feb;97(8):e9967.

9. Chien TW, Chow JC, Chang Y, Chou W. Applying Gini coefficient to evaluate the author research domains associated with the ordering of author names: A bibliometric study.Medicine (Baltimore). 2018 Sep;97(39):e12418.

10. Hopkins R. NBA injuries from 2010-2018(analyze injuries in the NBA).2019/6/20 retrieved at https://www.kaggle.com/ghopkins/nba-injuries-2010-2018/activity.

11. Fenner T, Harris M, Levene M, Bar-llan J. A novel bibliometric index with a simple geometric interpretation. PLoS One. 2018;13(7):e0200098.

12. Hirsch JE. An index to quantify an individual's scientific research output. Proc. Natl. Acad. Sci. U. S. A. 2005;102: 16569-165728.

Page $11 / 18$ 
13. Egghe L, Rousseau R, Van Hooydonk G. Methods for accrediting publications to authors or countries: Consequences for evaluation studies. J Am Soc Inform Sci. 2000;51(2):145-57.

14. Efron B. Bootstrap methods: Another look at the jackknife. The Annals of Statistics. 1979;7(1):1-26.

15. Pan RK, Fortunato S. Author Impact Factor: tracking the dynamics of individual scientific impact. Sci Rep. 2014;4:4880.

16. Huang MH, Chi PS. A Comparative Analysis of the Application of H-index, G-index, and A-index in Institutional-Level Research Evaluation. Journal of Library Information Studies. 2010;8(2):1-10.

17. Kalcioglu MT, Ileri Y, Ozdamar OI, Yilmaz U, Tekin M. Evaluation of the academic productivity of the top 100 worldwide physicians in the field of otorhinolaryngology and head and neck surgery using the Google Scholar h-index as the bibliometrics ranking system. J Laryngol Otol. 2018 Dec;132(12):1097-101.

18. Accredited Endovascular Surgical Neuroradiology Programs: Current Specialty Composition and Academic Impact Using the $\mathrm{h}$ Index.World Neurosurg 10.1016/j.wneu.2019.05.038

Waqas M, Shakir HJ, Shallwani H, Beecher JS, Rangel-Castilla L, Siddiqui AH, Levy El.Accredited Endovascular Surgical Neuroradiology Programs: Current Specialty Composition and Academic Impact Using the h Index.World Neurosurg. 2019 May 13. pii: S1878-8750(19)31312-9. doi: 10.1016/j.wneu.2019.05.038.

19. Jin BH, Liang LM, Rousseau R, Egghe L. The R- and AR-indices: Complementing the h-index. Chin Sci Bull. 2007;52:855-63.

20. Bornmann L, Mutz R, Hug SE, Deniel JD. A multilevel meta-analysis of studies reporting correlations between the h-index and 37 different h-index variants. Journal of Informetrics 201; 5 (3): 346-59.

21. Bluhmki T, Putter $\mathrm{H}$, Allignol A, Beyersmann J, COMBACTE-MAGNET consortium. Bootstrapping complex time-to-event data without individual patient data, with a view toward time-dependent exposures. Stat Med. 2019. doi:10.1002/sim.8177.

\section{Tables}

Table 1 Top 20 frequent injuries and illnesses observed in this study 


\begin{tabular}{llrrr}
\hline No. Injury/illness & Count & \multicolumn{3}{c}{ \% Cluster } \\
\hline 1 & sprained left ankle & 537 & 7.25 & 1 \\
\hline 2 & sprained right ankle & 314 & 4.24 & 2 \\
\hline 3 & illness & 257 & 3.47 & 3 \\
\hline 4 & sore left knee & 230 & 3.1 & 5 \\
\hline 5 & sore right knee & 198 & 2.67 & 4 \\
\hline 6 & concussion & 153 & 2.06 & 6 \\
\hline 7 & back spasms & 137 & 1.85 & 7 \\
\hline 8 & knee injury & 120 & 1.62 & 8 \\
\hline 9 & strained right hamstring & 110 & 1.48 & 9 \\
\hline 10 & sore lower back & 102 & 1.38 & \\
\hline 11 & strained left hamstring & 97 & 1.31 & \\
\hline 12 & strained left calf & 91 & 1.23 & \\
\hline 13 & right knee injury & 82 & 1.11 & \\
\hline 14 & flu & 80 & 1.08 & \\
\hline 15 & sore left foot & 80 & 1.08 & \\
\hline 16 & sore back & 73 & 0.98 & \\
\hline 17 back injury & 70 & 0.94 & \\
\hline 18 & sore right ankle & 69 & 0.93 & \\
\hline 19 & sore left ankle & 67 & 0.9 & \\
\hline 20 & bruised right knee & 60 & 0.81 & 11 \\
\hline
\end{tabular}

Table 2 Comparison of the retirement ages between groups using the bootstrapping method Characters Median SE Min. Max. Lower CI Upper CI $n$ Significance

\begin{tabular}{|c|c|c|c|c|}
\hline \multicolumn{5}{|l|}{ A.All-stars } \\
\hline injury & 33.940 .4532 .7534 .38 & 33.05 & $34.76 \quad 69$ & \\
\hline non-injury & $35.5 \quad 0.38 \quad 33.836 .72$ & 34.83 & 36.24170 & Yes \\
\hline \multicolumn{5}{|l|}{ B:Regulars } \\
\hline injury & 28.760 .4327 .5230 .01 & 27.93 & 29.59408 & \\
\hline non-injury & 29.370 .4528 .0631 .02 & 28.49 & $30.24 \quad 58$ & No \\
\hline
\end{tabular}

\section{Supplemental Information}

\section{Additional File 1:}

Xlsx file: study dataset

\section{Additional File 2:}

MP4 file: demonstrating how to draw dashboard in Excel 
http://www.healthup.org.tw/marketing/course/marketing/NBAinjury.mp4

\section{Additional File 3:}

Worst NBA Injuries of the 2018-2019 Season

https://www.youtube.com/watch?v=10oK9bNnQ8M

\section{Figures}

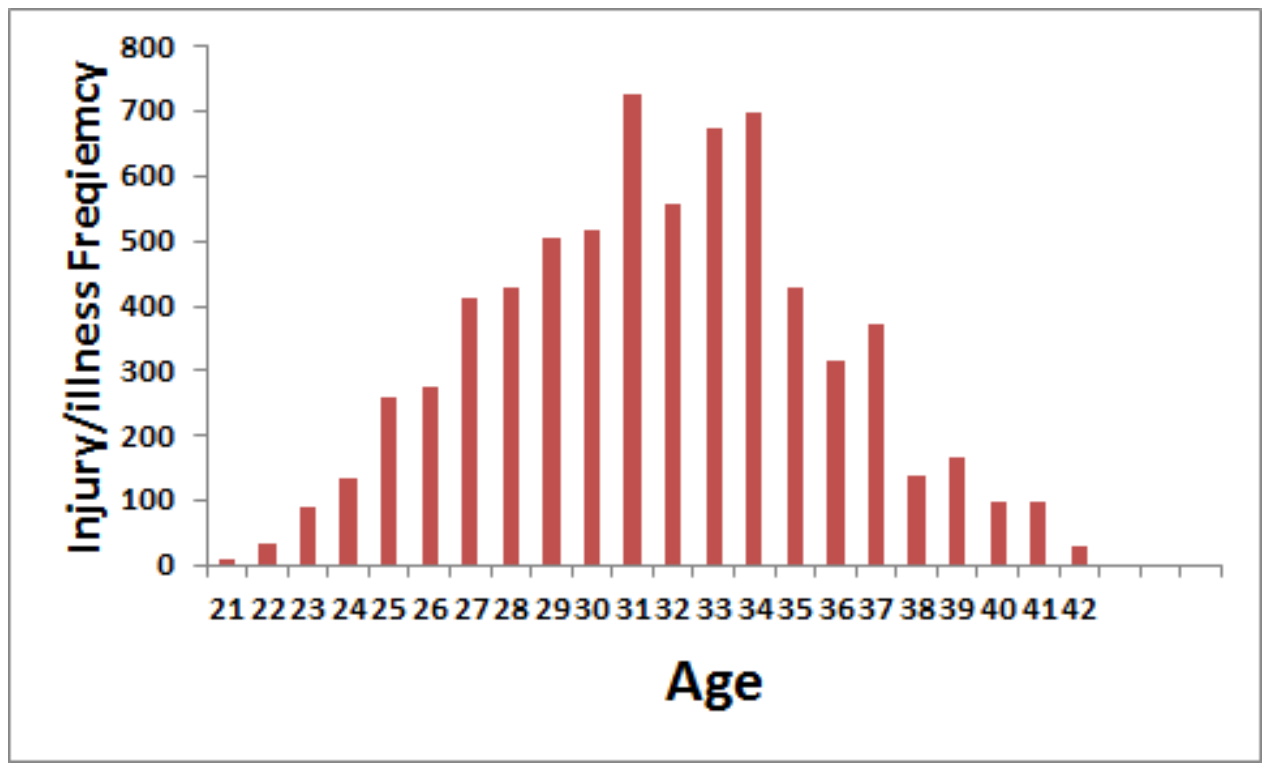

Figure 1

Injury/illness frequency distribution across different ages 


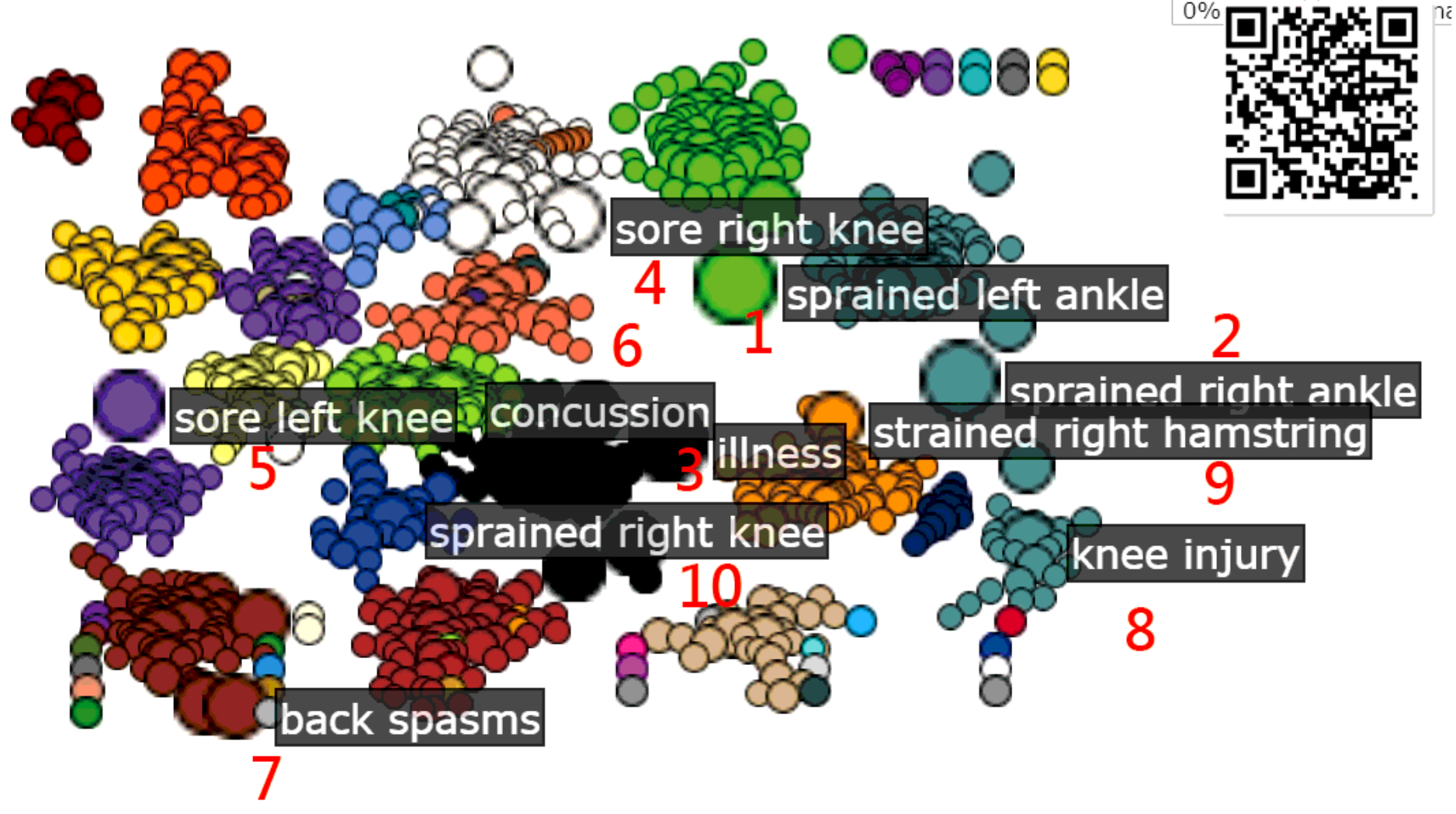

Figure 2

Main injuries of NBA players $(n=1174)$ 

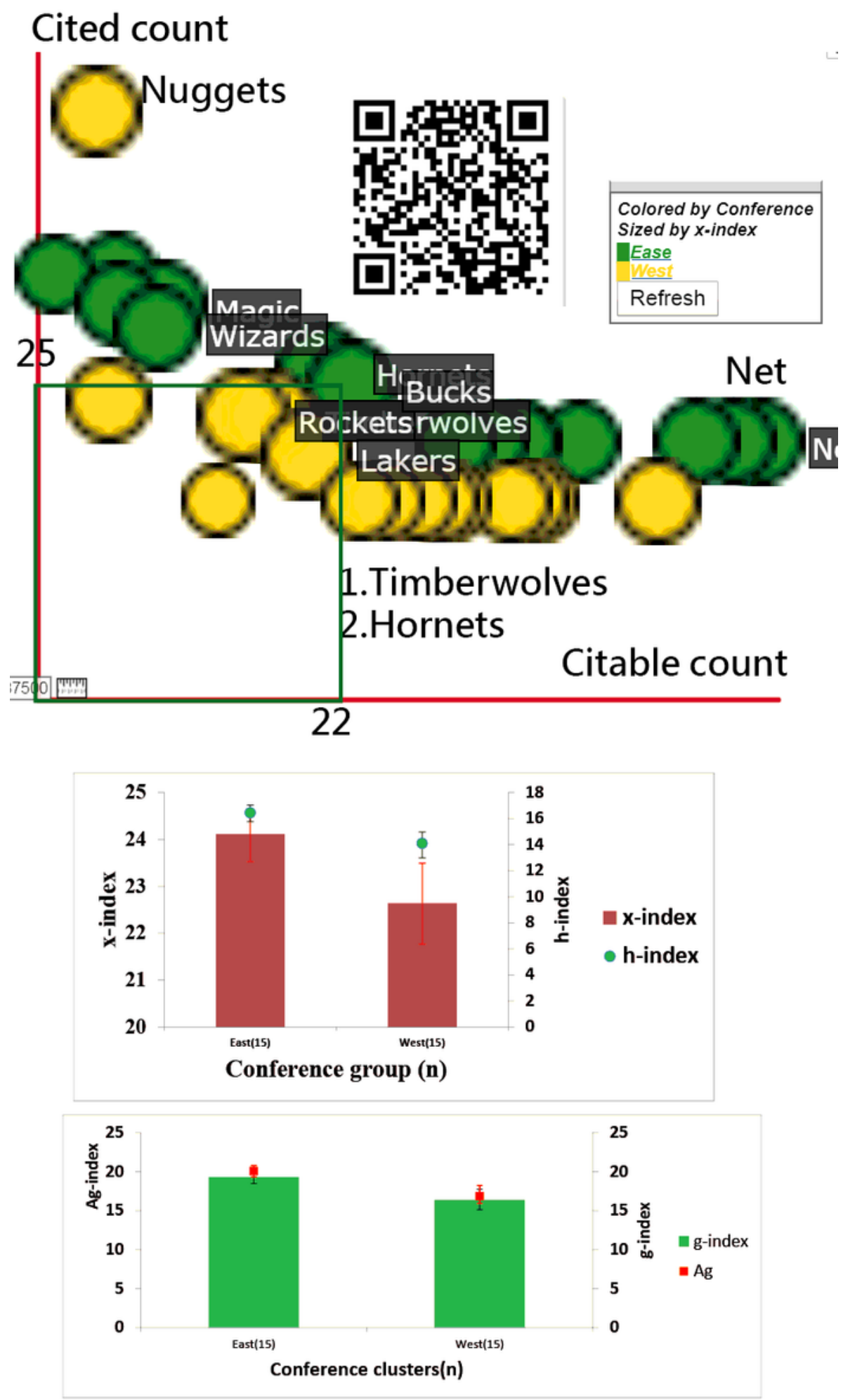

Figure 3

Most injured NBA teams and comparisons of metrics 

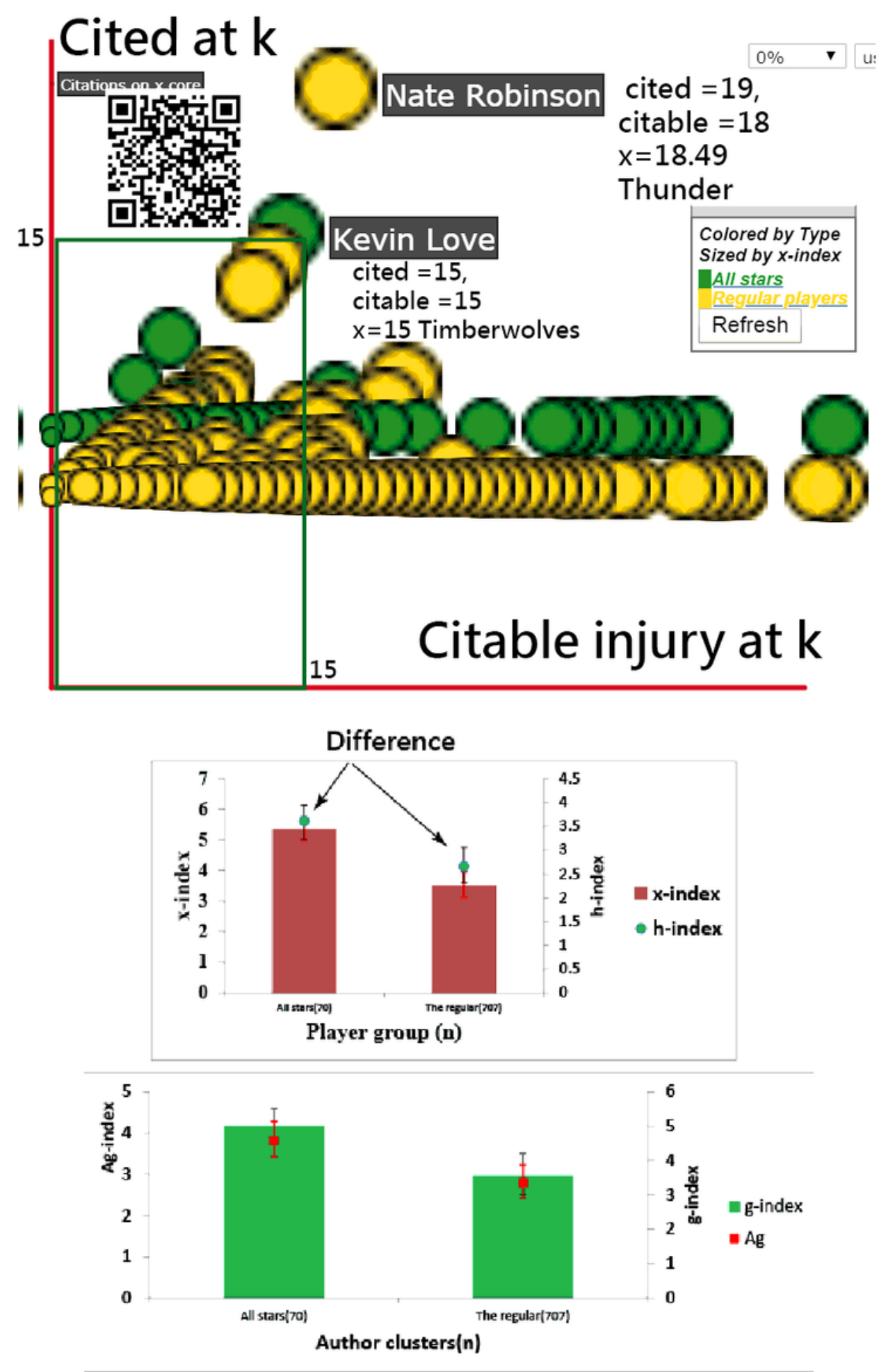

Figure 4

Most injured NBA players and comparisons of metrics

\section{Supplementary Files}

This is a list of supplementary files associated with this preprint. Click to download. 
- add3.txt

- add2.txt

- dataset.xlsx 